\title{
Cell-cycle-dependent regulation of androgen receptor function
}

\author{
Yulia Koryakina', Karen E Knudsen ${ }^{2}$ and Daniel Gioeli, ${ }^{1,3}$ \\ ${ }^{1}$ Department of Microbiology, Immunology, and Cancer Biology, University of Virginia, Jordan Hall Room 2-16, \\ 1300 Jefferson Park Avenue, PO Box 800734, Charlottesville, Virginia 22908, USA \\ ${ }^{2}$ Sidney Kimmel Cancer Center, Thomas Jefferson University, Philadelphia, Pennsylvania, USA \\ ${ }^{3}$ Cancer Center Member, University of Virginia, Charlottesville, Virginia, USA
}

Correspondence

should be addressed

to D Gioeli

Email

dgg3f@virginia.edu

\begin{abstract}
The androgen receptor $(A R)$ is a critical oncogene in prostate cancer (PCa) development and progression. In this study, we demonstrate cell-cycle-dependent regulation of AR activity, localization, and phosphorylation. We show that for three AR-target genes, androgenstimulated AR transactivation is highest during the G1 phase, decreased during S-phase, and abrogated during G2/M. This change in AR transactivation parallels changes in AR localization and phosphorylation. A combination of imaging techniques and quantitative analysis reveals nuclear AR localization during interphase and the exclusion of the majority, but not all, AR from chromatin during mitosis. Flow cytometry analyses using a phosphoS308 AR-specific antibody in asynchronous and chemically enriched G2/M PCa cells revealed ligand-independent induction of S308 phosphorylation in mitosis when CDK1 is activated. Consistent with our flow cytometry data, IP-western blotting revealed an increase in S308 phosphorylation in G2/M, and the results of an in vitro kinase assay indicated that CDK1 was able to phosphorylate the AR on S308. Pharmacological inhibition of CDK1 activity resulted in decreased S308 phosphorylation in PCa cells. Importantly, using a combination of anti-total AR and phospho-S308-specific antibodies in immunofluorescence experiments, we showed that the AR is excluded from condensed chromatin in mitotic cells when it was phosphorylated on S308. In summary, we show that the phosphorylation of the AR on S308 by CDK1 during mitosis regulates AR localization and correlates with changes in $A R$ transcriptional activity. These findings have important implications for understanding the function of $A R$ as an oncogene.
\end{abstract}

$\begin{aligned} & \text { Key Words } \\ & \text { - androgen receptor } \\ & \text { - cell cycle } \\ & \text { - phosphorylation } \\ & \text { - localization } \\ & \text { - mitosis }\end{aligned}$

Endocrine-Related Cancer (2015) 22, 249-264

\section{Introduction}

Inhibition of androgen receptor (AR) function is a standard therapy for the initial presentation of disseminated prostate cancer (PCa) (George \& Moul 2011). However, treatment almost invariably fails, leading to the emergence of castration-resistant prostate cancer (CRPC). The clinical importance of targeting AR function in CRPC is best illustrated by the FDA approval of abiraterone, a CYP17 inhibitor, and the anti-androgen, enzalutamide.
Unfortunately, the clinical benefit of these new therapies is not durable (Joseph et al. 2013). These observations further emphasize the importance of AR signaling in PCa development and progression. Only a thorough understanding of AR biology will provide novel insights into how to therapeutically target this critical driver of PCa.

The AR functions as a driver of G1 progression through cross-communication with the cell cycle

Published by Bioscientifica Ltd. 
machinery and regulation of transcription of genes that control the G1-S transition (Balk \& Knudsen 2008). Upon androgen withdrawal, PCa cells arrest in early G1 with hypo-phosphorylated RB suppressing E2F activity (Knudsen et al. 1998, Xu et al. 2006). Stimulation with androgen leads to the accumulation of cyclin D1 and activation of CDK4, which promotes phosphorylation of $\mathrm{RB}$ (Xu et al. 2006). Furthermore, AR-induced expression of p21 and degradation of p27 enhance $\mathrm{CycD} / \mathrm{CDK} 4$ and $\mathrm{CycE} / \mathrm{CDK} 2-$ dependent phosphorylation and inactivation of $\mathrm{RB}$ allowing the expression of E2F-target genes (Knudsen et al. 1998, Lu et al. 1999). Thus, androgeninduced alterations in CDK activity enable expression of genes critical for S-phase entry (Knudsen 2006).

A cross-talk between AR signaling and the cell cycle machinery is not limited to the effects of androgens on the G1-S transition as several components of the cell cycle machinery have been shown to modulate AR function. It was first noted, in a study using fibroblasts, that AR activity is regulated as a function of the cell cycle; the results of this study indicated that AR transcriptional activity is lowest at the G1/S transition, when cyclin D1 levels and CDK4 activity are at their peak (Martinez \& Danielsen 2002). Cyclin D1 represses AR transcriptional activity independently of CDK4 by directly binding the coactivator-binding-AR dimerization motif in the AR AF-1 (Knudsen et al. 1999, Reutens et al. 2001, Martinez \& Danielsen 2002, Petre et al. 2002). This interaction competes with AR coactivators such as p300/CAF and interferes with N/C-terminal AR interactions (Knudsen et al. 1999, Reutens et al. 2001, Burd et al. 2006). Thus, cyclin D1 can act in a negative feedback loop, attenuating AR activity. This cyclin D1 repression is disrupted at multiple levels in human tumors facilitating increased AR activity (Burd et al. 2006, Knudsen 2006, Comstock \& Knudsen 2007). Cyclin E has also been shown to associate with the AR AF-1 to enhance AR transcription independently of CDK2 (Yamamoto et al. 2000). In addition, CDK6 negates the ability of cyclin D1 to suppress AR function, and can serve to heighten AR activity independent of its kinase function (Lim et al. 2005). However, surprisingly little has been reported on the role of the AR in G2 or mitosis.

The effect of the cell cycle on AR protein expression has been examined in one study where it was suggested that AR protein expression is lost during mitosis and that the AR functions as a mitotic licensing factor (Litvinov et al. 2006). However, others have reported that the AR is bound to condensed chromatin during mitosis (Kumar et al. 2008). Thus, little is known about the AR during
G2/M and what is postulated about the AR during mitosis is conflicting.

In this study, we examined endogenous AR transcriptional activity, protein levels, localization, and phosphorylation during the cell cycle. We found that for a subset of AR-dependent genes, transcription is highest during the G1 phase of the cell cycle, reduced during S-phase, and essentially abrogated during G2/M. This change in transcription was not due to a reduction in AR levels during cell cycle progression. AR localization changes in mitotic cells compared with interphase cells. This change in AR localization and reduction in transactivation correlated with AR phosphorylation on S308 during mitosis. AR S308 phosphorylation occurs during mitosis coincident with peak CDK1 activity and pharmacological inhibition of CDK1 abrogated S308 phosphorylation. Moreover, CDK1 phosphorylated the $\mathrm{AR}$ on $\mathrm{S} 308$ in an in vitro kinase assay. Using a combination of phospho-S308-specific and anti-total AR antibodies, we showed that: i) only mitotic PCa cells express AR phosphorylated on S308; and ii) AR phosphorylated on S308 is excluded from chromatin. The data reported herein indicates that AR phosphorylation on S308 by CDK1 regulates AR localization during mitosis. We further propose that this CDK1-mediated change in AR localization may regulate AR transcriptional activity. These observations have important implications for understanding AR biology and elucidating possible mechanisms of PCa progression.

\section{Materials and methods}

\section{Cell culture and treatment conditions}

PCa cell lines LNCaP and C4-2 were cultured in T-Media supplemented with $5 \%$ fetal bovine serum (FBS) (Invitrogen). For experiments, cells were switched to phenol-red-free RPMI supplemented with 5\% charcoalstripped serum (CSS) (Invitrogen). PC3 and COS7 cell lines were maintained in DMEM with 5\% FBS. LHS cells were cultured in PrEBM media supplemented with growth factors (Lonza, Allendale, NJ, USA). RWPE-1 cells were maintained in KLM media supplemented with pituitary factors. The identity of our cell lines was verified by DNA fingerprinting using commercial kits containing multiple STR markers (DDC Medical, Fairfield, OH, USA). The cells were synchronized in $\mathrm{G} 2 / \mathrm{M}$ by aphidicolin-block/release and nocodazole block: cells were treated with $2 \mu \mathrm{g} / \mathrm{ml}$ aphidicolin for $24 \mathrm{~h}$, washed, and treated with $50 \mathrm{ng} / \mathrm{ml}$

Published by Bioscientifica Ltd 
nocodazole for $16 \mathrm{~h}$. For ImageStream experiments, cells were treated with aphidicolin and released for $12 \mathrm{~h}$.

\section{Cell sorting}

For protein analysis, growing cells were stained with $4 \mu \mathrm{g} / \mathrm{ml}$ Hoechst 3342 and collected for sorting. For RNA, cells were treated with $1 \mathrm{~nm}$ DHT for $2 \mathrm{~h}$, washed, collected, and fixed in cold methanol for $20 \mathrm{~min}$. Then cells were washed with PBS and stained with $4 \mu \mathrm{g} / \mathrm{ml}$ Hoechst 3342 at $4{ }^{\circ} \mathrm{C} \mathrm{O} / \mathrm{N}$. The cells were sorted into cell cycle compartments on the basis of DNA content using a Vantage flow cytometer. The sorted cells were centrifuged and cell pellets were subjected to lysis for protein or RNA isolation as previously described (Gordon et al. 2010, Whitworth et al. 2012).

\section{QRT-PCR}

qRT-PCR experiments were performed as previously described (Gordon et al. 2010, Whitworth et al. 2012). The primers were as follows: SNAI2, forward: $5^{\prime}$-CTCCATCTGACACCTCCT-3', reverse: 5'-ACTGTAGTCTTTCCTCTTCATC-3'; SGK1, forward: 5'-GGATGGGTCTGAACGAC TTT-3', reverse: 5'-GAAGGACTTGGTGGAGGAGA-3'; UBE2C, forward: 5'-TGGTCTGCCCTGTATGATGT-3', reverse: $5^{\prime}$-AAAAGCTGTGGGGTTTTTCC-3'; PSMB6, forward: 5'-CAAACTGCACGGCCATGATA-3', reverse: 5'-GAGGCATTCACTCCAGACTGG-3'; GUS, forward: 5'-CTCATTTGGAATTTTGCCGATT-3', reverse: $5^{\prime}$-CCGAGTGAAGATCCCCTTTTTA- $3^{\prime}$. The expression levels of analyzed genes were normalized to those of the housekeeping genes proteasome subunit beta type-6 (PSMB6) and beta-glucuronidase (GUS).

\section{Flow cytometry}

For flow cytometry experiments using anti-phospho Histone-H3 (Cell Signaling, Danvers, MA, USA) and anti-AR antibodies, cells were fixed in freshly prepared $4 \%$ $(\mathrm{w} / \mathrm{v})$ paraformaldehyde (PFA), $\mathrm{pH} 7.2$, for $15 \mathrm{~min}$ and then permeabilized with cold methanol for $20 \mathrm{~min}$. The cells were blocked using a blocking buffer containing 5\% goat serum and then incubated with mix of mouse anti-phospho Histone-H3 (Cell Signaling) and rabbit anti-AR (antigen, first 20 N-terminal amino acids (Gordon et al. 2010)) primary antibodies followed by separate incubations with mix of secondary goat fluorescein (FITC)-conjugated antimouse antibody (Jackson ImmunoResearch, West Grove, PA, USA) and goat R-phycoerythrin (PE)-conjugated antirabbit antibody (Invitrogen, Molecular Probes). The alternative anti-AR antibody used in flow cytometry studies was mouse antibody from BD Pharmingen (part of BD Biosciences, San Jose, CA, USA). DNA was stained with TO-PRO3 iodide (Invitrogen). Primary rabbit antiphospho-S308 antibody was from Santa Cruz. Data were collected using a BD FACSCalibur flow cytometer (BD Biosciences). FITC and PE fluorescence was detected in the FL1 and FL2 channels, respectively, and TO-PRO-3 fluorescence was detected in the FL4 channel, with CellQUEST Pro Software (Becton-Dickinson, Franklin Lakes, NJ, USA). Data were analyzed using FlowJo 8.6.6 Software. For the ImageStream experiments, samples were stained similarly as for flow cytometry and imaged on an ImageStream imaging cytometer (Amnis, EMD Millipore, Billerica, MA, USA ) with data analysis using IDEAS 4.0 Software.

\section{Fluorescence microscopy}

For indirect immunofluorescence microscopy, cells were grown on glass coverslips in six-well dishes, treated with $50 \mathrm{ng} / \mathrm{ml}$ nocodazole for $16 \mathrm{~h}$, and fixed in freshly prepared $4 \%$ PFA for $20 \mathrm{~min}$. The samples were rinsed with PBS, blocked and permeabilized in blocking/antibody dilution buffer containing 5\%-normal goat serum and $0.3 \%$-Triton $\mathrm{X}$ in PBS for $1 \mathrm{~h}$. The samples were incubated with anti-AR or anti-phospho-S308 AR primary antibodies followed by secondary goat Alexa Flour 594-conjugated anti-rabbit (Invitrogen, Molecular Probes) or secondary goat FITC-conjugated anti-mouse antibody (Jackson ImmunoResearch). Anti-AR antibodies used were a rabbit antibody developed against the N-terminal domain (Gordon et al. 2010), and mouse antibodies from Santa Cruz (AR441) and BD Pharmingen. Optimal dilutions of the antibodies were determined in titration experiments. The samples were mounted in Vectashield mounting medium containing 4',6-diamidino-2-phenylindole (DAPI) for nuclear staining (Vector Laboratories, Inc., Burlingame, CA, USA) and visualized by confocal microscopy using the LSM510. A Zeiss Plan-Apo $40 \times$ $1.40 \mathrm{NA}$ oil immersion objective was used for image acquisition. Co-localization of AR and DNA was quantified by calculating the percentage of signal overlap as described previously (Koryakina et al. 2012).

\section{In vitro kinase assay and western blots}

The in vitro kinase assays and western blots were performed as described previously (Gordon et al. 2010, Whitworth et al. 2012) and analyzed using an Odyssey (Licor, Lincoln, $\mathrm{NE}$, USA) imaging system.

Published by Bioscientifica Ltd 


\section{Statistical methods}

Differences between groups were analyzed using one-way ANOVA. Where multiple groups were compared using ANOVA, a post hoc Tukey's test was used to enable multiple comparisons between groups.

\section{Results}

\section{AR transcriptional activity changes during the cell cycle}

AR transactivation of endogenous genes was assessed by performing qRT-PCR analyses of the AR target genes TMPRSS2, SGK1, and SNAI2 of LNCaP cells sorted into the G1, S-early, S-late, and G2/M cell cycle compartments. LNCaP cells were sorted on the basis of DNA content (Fig. 1A). We found that androgen-dependent transcription decreases at these AR target genes during the cell cycle (Fig. 1). Transcription of SGK (SGK1), SNAI2, and TMPRSS2 increased in response to DHT with the greatest induction during G1, decreased during S-phase, and for SGK1 and SNAI2, it was essentially abrogated in G2/M. This decrease in transcription was not due to a global effect on transcription; UBE2C, which encodes a ubiquitin-conjugating ligase necessary for degradation of mitotic cyclins, demonstrated the inverse pattern of expression where UBE2C expression was minimal in G1, increased in S-phase, and showed the highest levels of expression in $\mathrm{G} 2 / \mathrm{M}$, consistent with its function in mitosis (Fig. 1E). In addition, the expression of the housekeeping gene PSMB6 encoding the proteasome subunit, beta type 6 (and the housekeeping gene GUS, data not shown) did not change during the cell cycle (Fig. 1F).

\section{AR levels do not change during the cell cycle}

We initially proposed the hypothesis that the change in AR transcriptional activity could be due to changes in AR levels during the cell cycle because a results from a previous study indicated that the AR is degraded during mitosis (Litvinov et al. 2006). We performed a series of flow cytometry experiments using two different anti-AR antibodies targeting different epitopes. To distinguish cell cycle compartments, DNA was stained with TO-PRO3. Histone $\mathrm{H} 3$ phosphorylation on Ser10 is necessary for chromosome condensation during mitosis (Van Hooser et al. 1998) and was used as a mitotic marker in our studies. Dualparametric analysis of AR fluorescence and DNA fluorescence did not reveal differences in the AR levels during the cell cycle in LNCaP and C4-2 cells (Fig. 2A and B). Gating on mitotic phospho-histone H3-positive and interphase phospho-histone H3-negative populations to analyze AR levels in mitotic and interphase cells (Fig. 2C), we found that $\sim 0.7-1 \%$ of asynchronous LNCaP cells expressed phospho-histone H3. AR levels in interphase and mitotic cells were very similar as shown in Fig. $2 \mathrm{D}$ and $\mathrm{E}$ for LNCaP and C4-2 cells respectively.
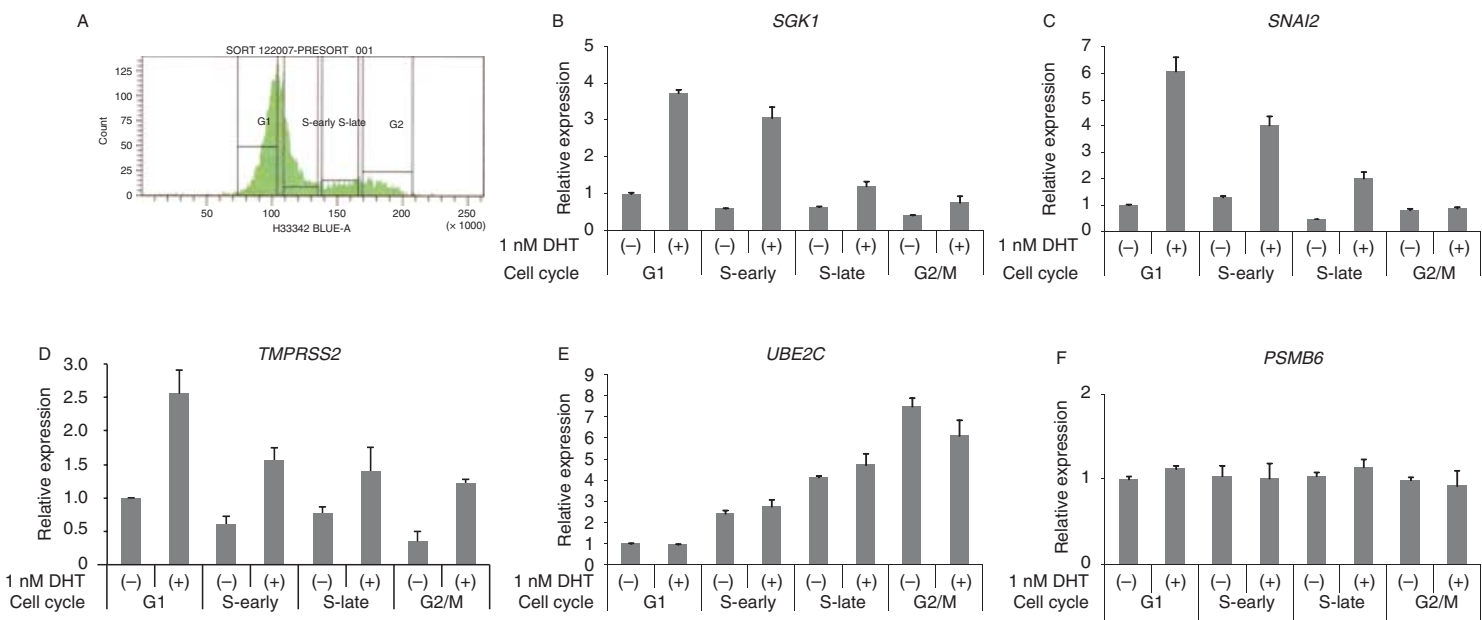

\section{Figure 1}

Regulation of AR target genes during the cell cycle. LNCaP cells were grown in T-Media supplemented with 5\% FBS. Media was switched to phenolred-free RPMI with 5\% CSS. The cells were treated with either $1 \mathrm{~nm}$ DHT or vehicle, fixed, stained with Hoechst 3342, and sorted into cell cycle compartments by flow cytometry on the basis of DNA content. The expression of AR target genes in different cell cycle compartments was analyzed by qRT-PCR after cell sorting. (A) Histogram shows gating on Printed in Great Britain
(C) 2015 Society for Endocrinology cell cycle compartments during flow sorting on the basis of DNA content. AR-dependent genes SGK1 (B), SNAI2 (C), and TMPRSS2 (D) showed cellcycle-specific regulation. (E) Transcription of UBE2C was increased during G2/M and was not changed in response to DHT. (F) Transcription of the housekeeping gene PSMB6 was uniform throughout the cell cycle. A full colour version of this figure is available at http://dx.doi.org/10.1530/ ERC-14-0549.

Published by Bioscientifica Ltd. 

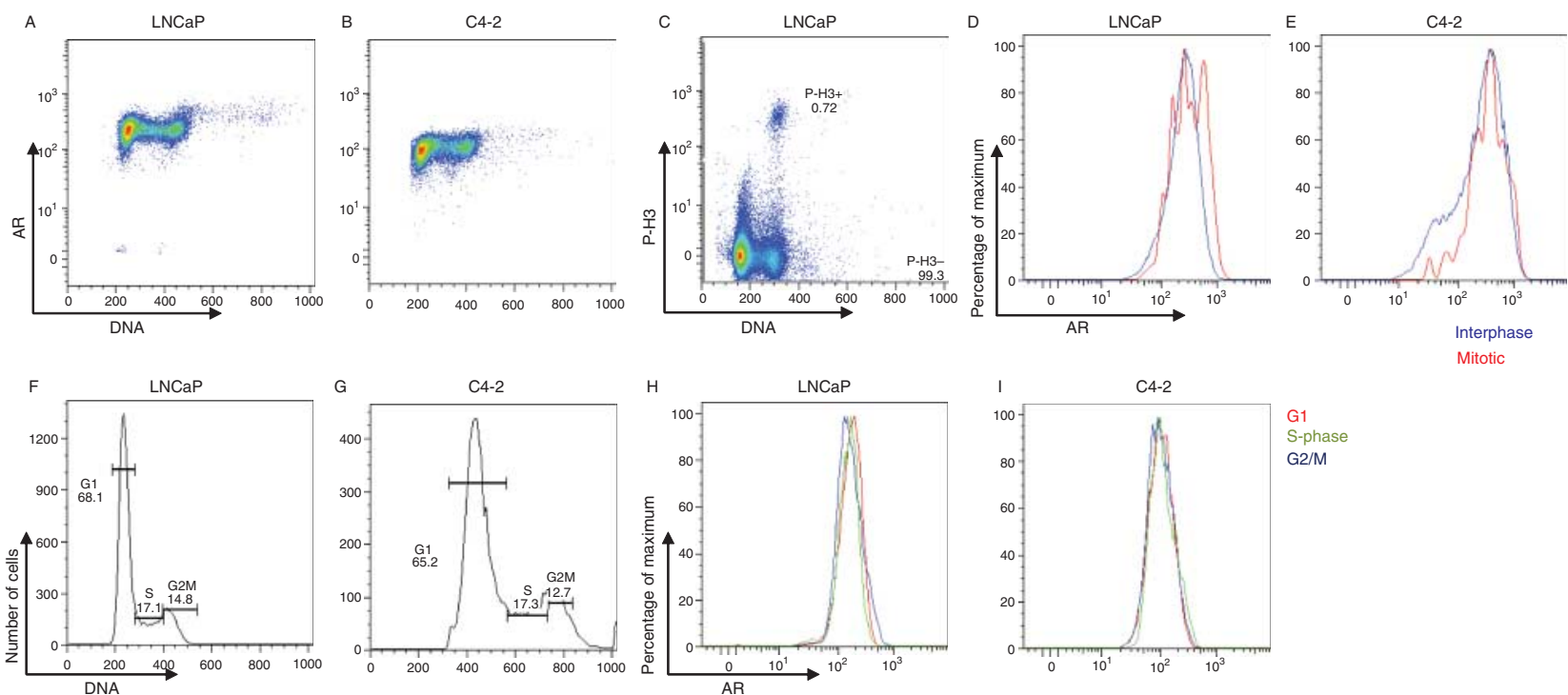

\section{Figure 2}

Analyses of AR protein levels during the cell cycle by flow cytometry. The cells were fixed and stained with two different anti-AR antibodies. Phospho-histone $\mathrm{H} 3$ staining was used to identify the mitotic population. DNA was stained with TO-PRO3. Dual-parametric analyses of AR and DNA fluorescence in LNCaP (A) and C4-2 (B) cells reveal similar levels of AR during the cell cycle. (C) Dual-parametric fluorescence analysis of phosphohistone-H3 signal versus DNA shows mitotic phospho-histone H3-positive, and interphase phospho-histone H3-negative cells in LNCaP cells.

AR levels during the cell cycle were analyzed in further detail by gating on cell populations in G1, S, and G2/M phases of the cell cycle using DNA staining (Fig. 2F and G). As shown in Fig. 2H and I, AR levels in G1, S, and G2/M phases overlap in both PCa cell lines. Using a second antiAR antibody, AR levels were similar in G1, S, and G2/M phases in both PCa cell lines (Supplementary Figure S1, see section on supplementary data at the end of this article). The specificity of these anti-AR antibodies was confirmed by analyzing total AR expression in AR-positive and AR-negative cell lines; AR-positive LNCaP and C4-2 demonstrated high levels of signal for AR fluorescence, whereas the AR-negative PC3 and LHS, as well as COS7 cells, had left-shifted peaks for AR fluorescence that were set as background fluorescence (Supplementary Figure S1). Overall, our flow cytometry analysis revealed that AR levels do not change during the cell cycle.

\section{AR localization is different in interphase and mitotic cells}

AR localization in PCa cells was assessed by confocal microscopy in cells enriched in mitosis. AR demonstrated predominantly nuclear staining in interphase cells. In mitotic cells marked by the characteristic DNA
Histograms in (D) and (E) show AR levels in mitotic (red) and interphase (blue) cells gated on phospho-histone $\mathrm{H} 3$ staining in LNCaP and C4-2 cells respectively. Histograms in (F) and (G) show gating on G1, S-phase, and G2/M cell cycle compartments based on DNA staining in LNCaP (E) and C4-2 (F) cells. Histograms illustrate AR protein levels during G1 (red), S-phase (green), and G2/M (blue) in LNCaP (H) and C4-2 (I) cells using anti-AR antibody directed against the first 21 amino acids of the AR (AR21).

condensation of condensed chromosomes, a substantial portion of the AR was excluded from chromosomes. LNCaP and C4-2 cells had similar patterns of AR staining in interphase and mitotic cells (Fig. 3A and B). AR-negative prostate epithelial cells, RWPE-1, did not stain for AR, further confirming the specificity of the AR antibody (Fig. 3C).

To further validate the change in AR localization in mitotic cells observed by confocal microscopy and quantify this observation, we analyzed AR localization during the cell cycle in LNCaP and C4-2 cells using the ImageStream imaging flow cytometer. This technology combines advantages of flow cytometry and epifluorescence microscopy enabling quantitative analysis of protein localization. In asynchronous LNCaP cells, $0.7-21 \%$ of cells are found in mitosis (Fig. 2C). To enrich cells in mitosis without the confounding variable of chemical arrest, cells were synchronized in S-phase with aphidicolin and then released so that the cells were free of chemical modifiers when progressing through mitosis (Fig. 4A). Similar to the results we observed in our confocal studies, AR staining was predominantly nuclear in interphase cells (Fig. 4C and D) and was largely excluded from mitotic chromosomes (Fig. 4E). The specificity of the AR staining was confirmed

Published by Bioscientifica Ltd. 

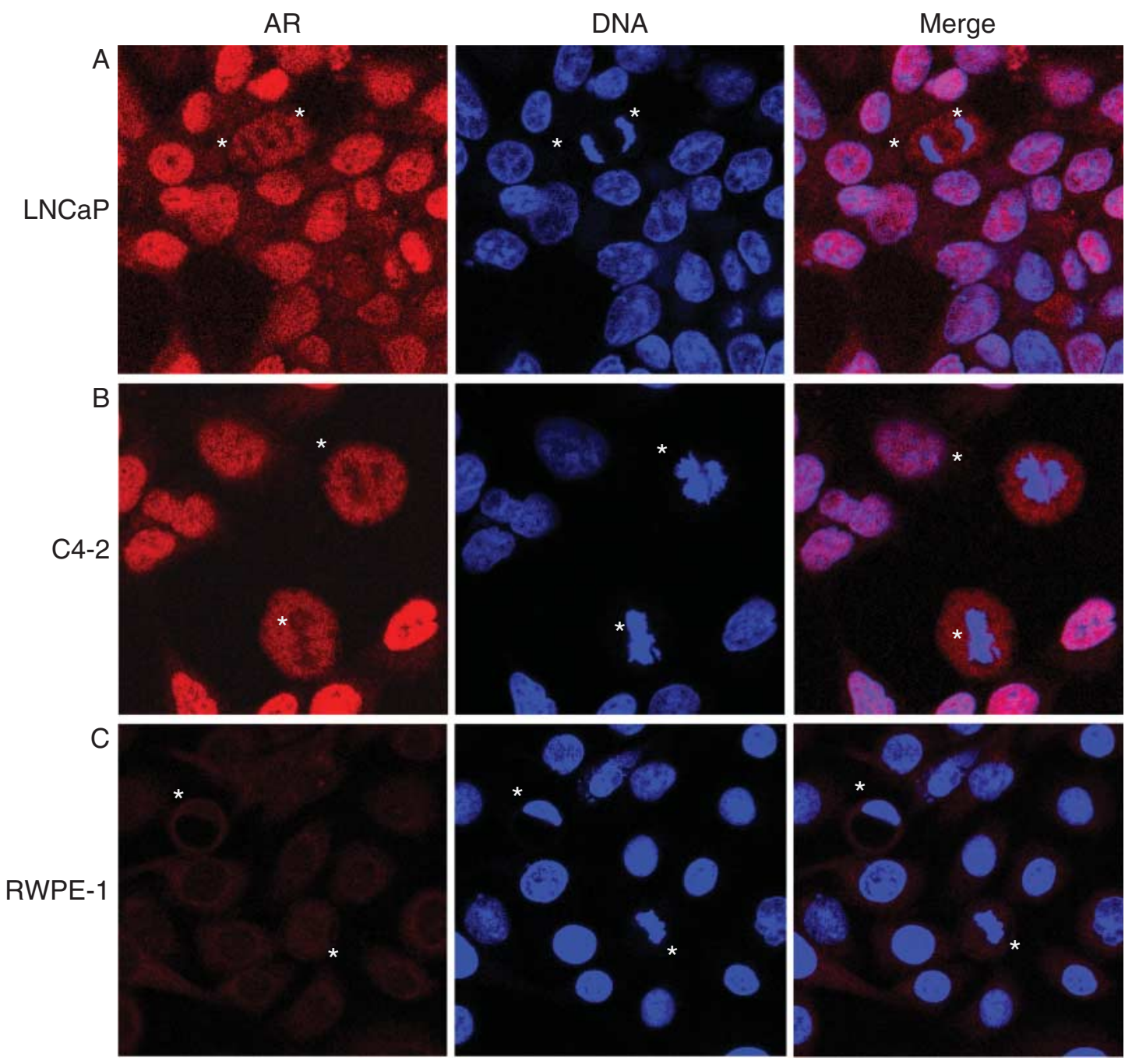

Figure 3

AR protein localization in interphase and mitotic prostate cancer cells by confocal microscopy. Cells were grown on cover slips, enriched in G2/M by nocodazole $(50 \mathrm{ng} / \mathrm{ml})$ treatment for $16 \mathrm{~h}$, fixed, and processed for indirect immunofluorescence. AR localization was analyzed using AR21 (red). DNA

by the absence of staining in the AR-negative cell line, LHS (Supplementary Figure S2A, see section on supplementary data at the end of this article).

To quantitatively assess AR localization with mitotic chromosomes, we examined similarity scores, which reflect the extent to which AR staining coincides with DNA staining. A positive similarity score reflects high co-localization, whereas a low or negative similarity score indicates that the AR does not co-localize with mitotic chromosomes. For example, the similarity score for phospho-histone H3 and DNA is $2.8 \pm 0.02$ (Supplementary Figure S2B, see section on supplementary data at the end of this article). This score provides an example of a high was stained with DAPI. Images were acquired using confocal microscopy (40 $\times$ objective). (A) LNCaP, (B) C4-2, and (C) RWPE-1 cells (negative control for AR staining). Mitotic cells are indicated by asterisks.

co-localization value. The $\beta 2$-adrenergic receptor is a G-protein-coupled receptor localized on the cell surface in the absence of $\beta$-agonist (Koryakina et al. 2012). Thus, this protein provides an example of a negative co-localization with DNA and a low similarity score; the similarity score for the $\beta 2$-adrenergic receptor and DNA is $-0.43 \pm$ 0.01 (Supplementary Figures S2C and S2D).

Similarity scores of AR and DNA were determined for three PCa cell cycle populations: mitotic cells, and two interphase populations: G1 + S-early and S-late $+\mathrm{G} 2$ cells. For interphase populations, gating was based on DNA content (Fig. 4B). In interphase cells, the AR shows nuclear staining (Fig. 4C and D) and high co-localization with

Published by Bioscientifica Ltd 


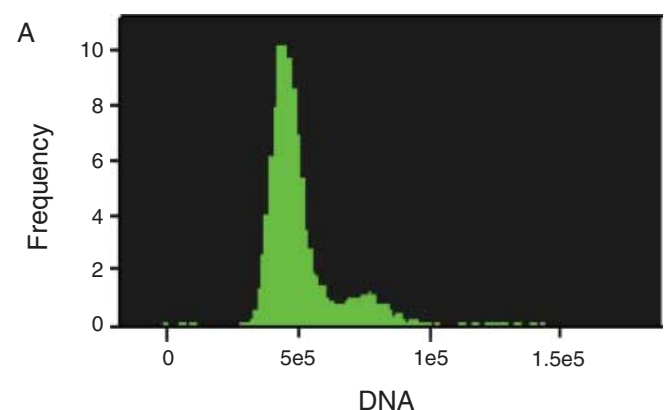

BF

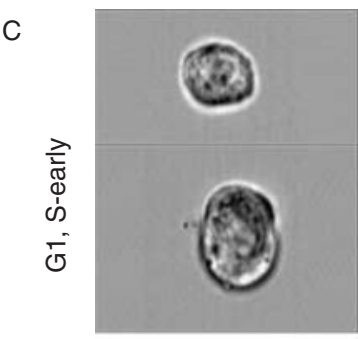

D

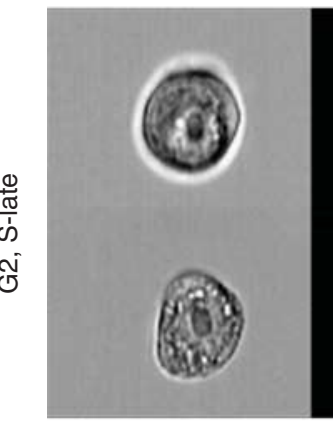

E
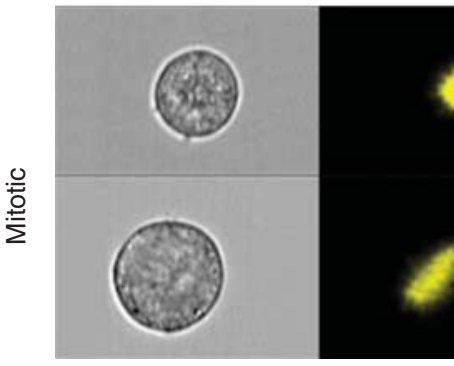

B

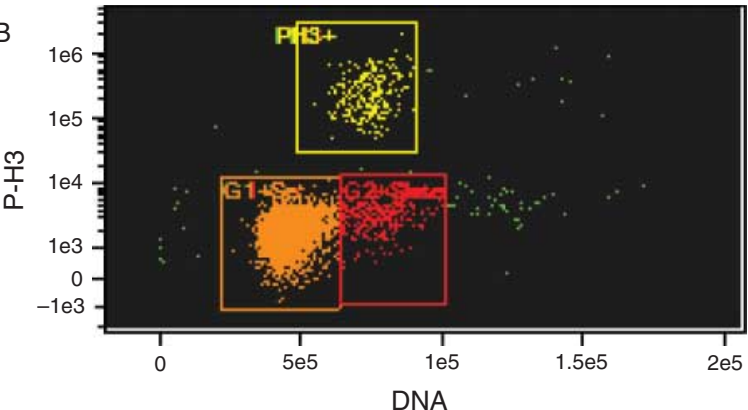

AR

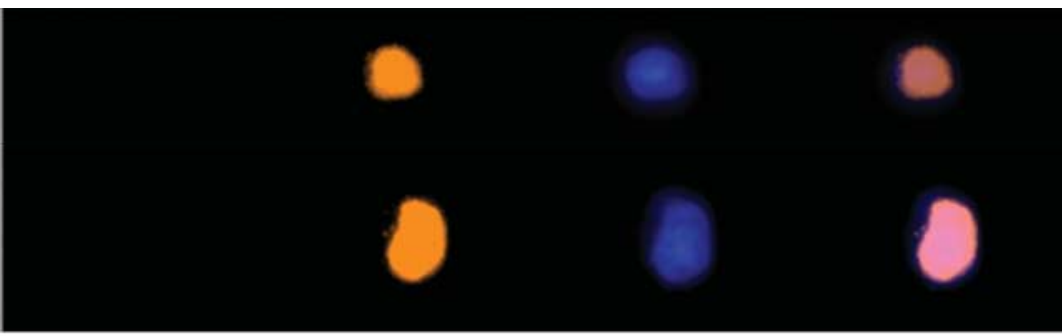

C4-2

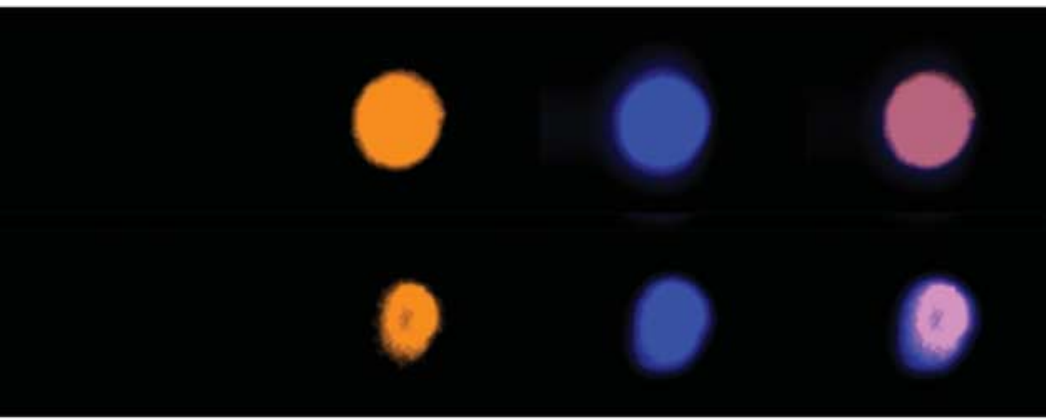

LNCaP

C4-2

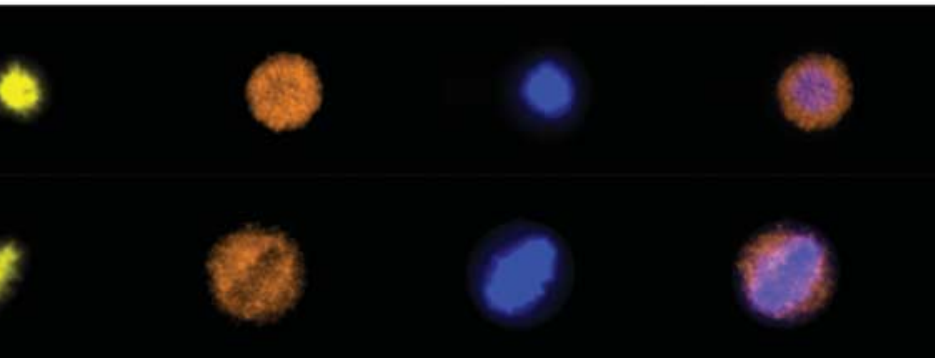

LNCaP

C4-2
F

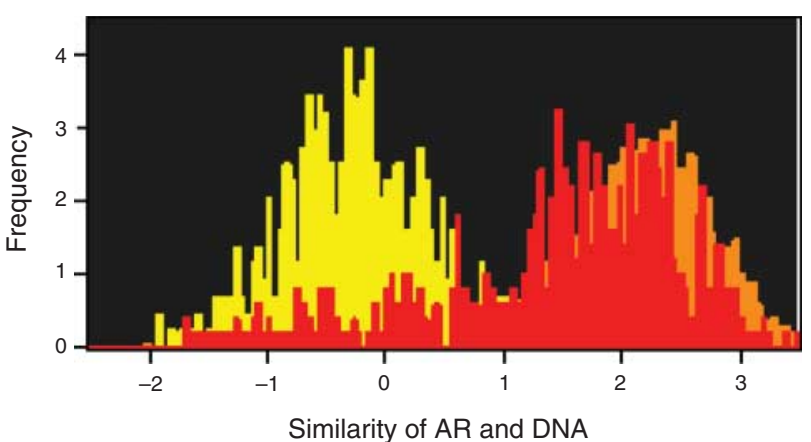

\begin{tabular}{|l|l|}
\hline $\begin{array}{l}\text { Cell cycle } \\
\text { compartment }\end{array}$ & $\begin{array}{l}\text { Similarity of AR } \\
\text { and DNA }\end{array}$ \\
\hline G1 + S-early & $2.55 \pm 0.27$ \\
\hline S-late + G2 & $2.05 \pm 0.21$ \\
\hline Mitotic & $-0.26 \pm 0.05$ \\
\hline
\end{tabular}

(C) 2015 Society for Endocrinology Printed in Great Britain 
DNA. The two interphase populations had similarity scores of $2.55 \pm 0.27$ and $2.05 \pm 0.21$ for G1+S-early and S-late $+\mathrm{G} 2$ respectively (Fig. $4 \mathrm{~F}$ and $\mathrm{G}$ ). These values are close to the similarity score for the positive control, phospho-histone H3 $(2.8 \pm 0.02)$. For the G1+S-early population, $98.8 \%$ of the cells showed AR co-localization with DNA while the S-late $+\mathrm{G} 2$ population showed 88.8\%; this reduced percentage is probably due to the redistribution of the AR from chromatin occurring in late G2 as cells are entering mitosis. In mitotic cells, the similarity score of the AR and DNA was strongly leftshifted in $95.6 \%$ of the cells, yielding a negative similarity score of $-0.26 \pm 0.05$ (Fig. $4 \mathrm{~F}$ and G). This similarity score of the AR and mitotic chromosomes was similar to that of the negative control, $\beta 2$-adrenergic receptor (Supplementary Figure S2C). Similar data were obtained with C4-2 cells (not shown). These results indicate that AR localization changes in mitotic when compared with interphase cells. In interphase cells, the AR is nuclear and co-localized with DNA whereas in mitotic cells the AR is predominately excluded from chromosomes (Fig. 4).

We next quantified the AR fluorescence intensity during each stage of mitosis to examine AR levels. The variation in $\mathrm{AR}$ fluorescence intensity for each stage of mitosis was only $5 \%$ from the AR fluorescence intensity of all mitotic cells. This indicates that AR levels do not change during mitosis and validates our previous observations that AR levels do not change during the cell cycle (Fig. 2 and Supplementary Figure S1).

\section{Hormone does not affect AR localization in mitosis}

The effects of a hormone on AR localization during mitosis was assessed. LNCaP and C4-2 cells were treated with $1 \mathrm{nM}$ DHT for $2 \mathrm{~h}$ and analyzed by ImageStream. In both cell lines, DHT treatment for $2 \mathrm{~h}$ did not significantly affect AR localization relative to DNA in mitotic cells. All $P$ values were $>0.05$ for all comparisons of AR and DNA co-localization in untreated versus hormone treated populations;
$P=0.56$ for $(-)$ versus $(+)$ DHT in Mitotic LNCaP; $P=0.86$ for $(-)$ versus $(+)$ DHT in Mitotic C4-2 (Supplementary Figure S3, see section on supplementary data at the end of this article).

\section{AR is excluded from chromosomes during all phases of mitosis}

Our data presented above indicates that AR protein levels are preserved during mitosis and that AR localization changes leading to AR exclusion from DNA. To analyze this further, we examined AR localization during all phases of mitosis. To isolate cells in specific phases of mitosis we used an algorithm that analyzes the shape of condensed chromosomes (Güttinger et al. 2009). The cells in different mitotic phases: prophase, metaphase, anaphase, and telophase, were isolated on the basis of the relationship between the width of chromosomes and the aspect ratio (ratio of width to length) of the phospho-histone H3 signal (Fig. 5A). Although the majority of mitotic cells were either in prophase or metaphase, we were able to isolate cells in all mitotic phases, including anaphase and telophase (Fig. 5B, C, D, and E). In all mitotic phases, the AR was present and largely excluded from DNA. These results further indicate that during mitosis the AR is predominately excluded from mitotic chromosomes.

\section{AR is phosphorylated on S308 in mitosis}

The AR is phosphorylated at multiple sites with different functional consequences, including regulation of AR localization (Koryakina et al. 2014). CDK1 is activated during mitosis, associates with the AR (Chen et al. 2006, Gordon et al. 2010) and regulates AR S81 phosphorylation (Chen et al. 2006, 2012). In addition to S81, the AR sequence contains multiple CDK1 consensus phosphorylation sites including a full site at S308. To directly test whether CDK1 can phosphorylate the AR on S308, we performed an in vitro kinase assay. CDK1, but not CDK5 or CDK9, phosphorylates AR S308 (Fig. 6A). When S308 phosphorylation is examined

\section{Figure 4}

AR localization is different in interphase and mitotic prostate cancer cells. LNCaP cells were enriched in mitosis by aphidicolin block-release: cells were treated with aphidicolin for $24 \mathrm{~h}$, washed, and released for $12 \mathrm{~h}$. Cells were fixed, stained for total AR, phospho-histone-H3, and DNA, and analyzed by using ImageStream. (A) Histogram showing cell cycle profile for LNCaP cells subjected to analysis. (B) Dual-parameter fluorescence analysis of phosphohistone $\mathrm{H} 3$ and DNA showing gating strategy for isolating cell cycle populations based on DNA content and the presence of the mitotic marker. Yellow: phospho-histone-H3-positive mitotic cells; red and orange: phospho-histone-H3-negative cells; orange: $\mathrm{G} 1$ and S early; and red: S late and G2/M. (C, D, and E) Representative images of LNCaP and C4-2 cells in
(C) 2015 Society for Endocrinology Printed in Great Britain each cell cycle compartment are shown. Note that only mitotic cells are positive for phospho histone-H3. (F) Representative graphical illustration of AR and DNA similarity scores in three populations: mitotic, G1 and $S$ early, and S late, and G2 cells. Color-coding is as in B. (G) Table showing similarity scores of AR and DNA in cell cycle compartments. Values shown are mean \pm S.E.M. from four independent experiments. The similarity scores are significantly different for $\mathrm{G} 1+\mathrm{S}$ early and mitotic populations $(P=0.0002)$ and $\mathrm{S}$ late $+\mathrm{G} 2$ and mitotic populations $(P=0.0002)$. Similarity scores for $\mathrm{G} 1+\mathrm{S}$ early and $\mathrm{S}$ late $+\mathrm{G} 2$ are not significantly different $(P=0.24)$. Post hoc Tukey's test was used.

Published by Bioscientifica Ltd. 


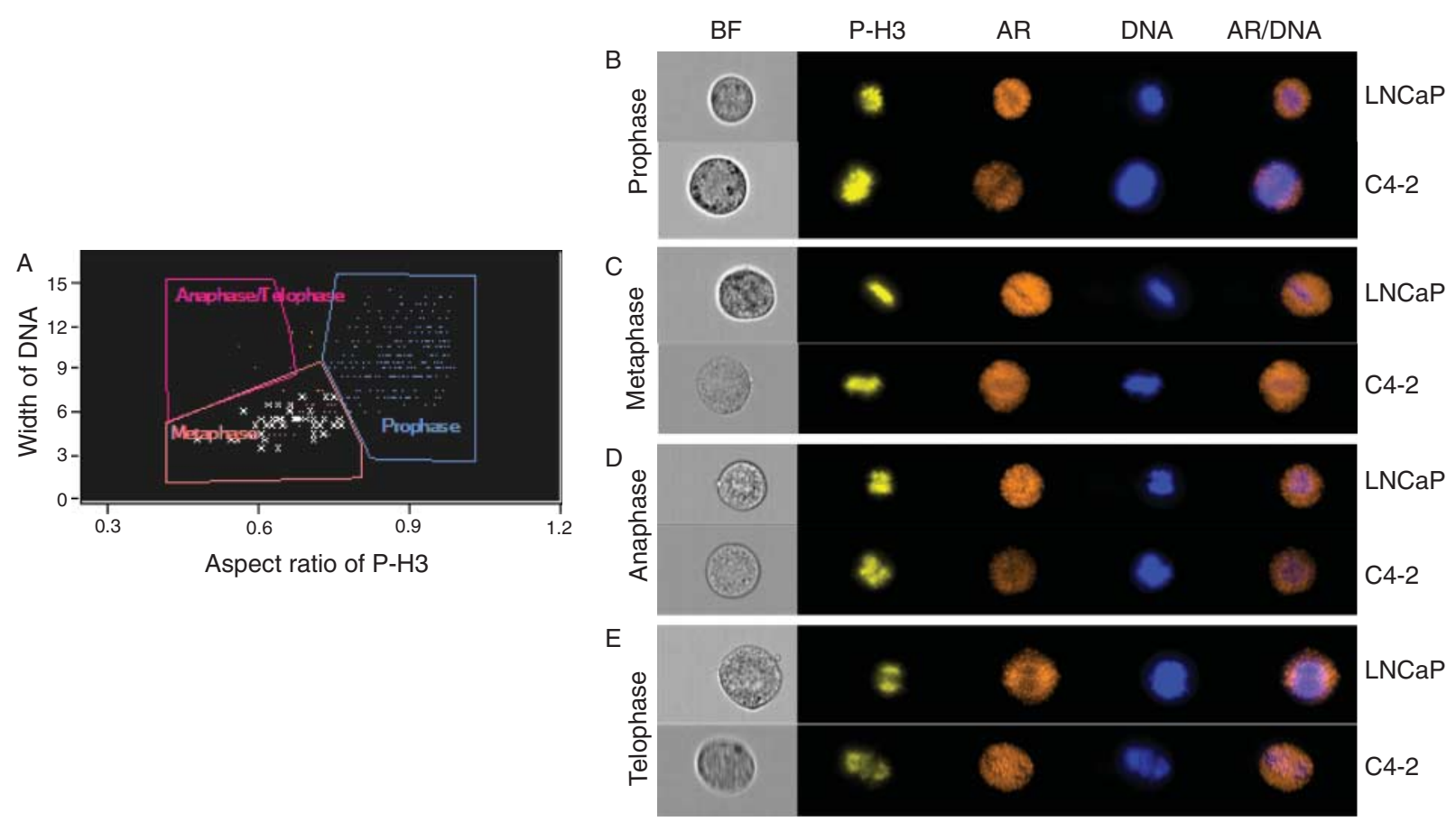

\section{Figure 5}

AR localization during mitosis. LNCaP and C4-2 were enriched in mitosis as previously described. Cells in different mitotic phases were isolated for analysis based on the aspect ratio of the phospho-histone $\mathrm{H} 3$ signal and

in asynchronous LNCaP cells sorted into G1, S-early, S-late, and G2/M groups, peak S308 phosphorylation is observed in G2/M, consistent with peak CDK1 activity and an increase in cyclin B levels (Fig. 6B).

To further explore the cell cycle regulation of AR S308, a series of flow cytometry experiments using asynchronous and G2/M-enriched LNCaP and C4-2 PCa cells was performed. The specificity of the phospho-specific S308 antibody was confirmed by analyzing AR-negative prostate cell lines and COS7 cells transfected with Flag-tagged WT and S308A mutant AR (Supplementary Figure S4, see section on supplementary data at the end of this article). A strong signal was observed in cells expressing WT AR, whereas no signal was seen in cells expressing S308A in COS7 cells expressing equivalent amounts of WT and S308A variants. AR-negative cell lines, LHS and PC3, did not have a phospho-S308 signal, further confirming the specificity of the phospho-S308 antibody.

The DNA profile for asynchronous LNCaP cells is shown in Fig. 7A. A small fraction of asynchronous LNCaP cells displayed AR S308 phosphorylation (Fig. 7B). All cells positive for phospho-S308 were in G2/M, whereas phospho-S308-negative cells were found during all stages of the cell cycle (Fig. 7B, C, and D). Similar results were width of DNA as shown in (A). Representative images of LNCaP and C4-2 cells in prophase (B), metaphase (C), anaphase (D), and telophase (E) are shown.

obtained in C4-2 cells (Fig. 7 E, F, and G). To confirm that S308 phosphorylation occurs during G2/M, we analyzed $\mathrm{LNCaP}$ cells enriched in G2/M. There were $60 \%$ of LNCaP cells enriched in G2/M (Fig. 7H) and a 40\% increase in the phospho-S308 signal (Fig. 7I). All phospho-S308-positive cells were localized in G2/M (Fig. 7J and K). To control for any potential confounding effects of chemical enrichment, we used an alternative approach where the dynamics of S308 phosphorylation was analyzed in cells synchronized in S-phase by aphidicolin and then released to progress through G2/M. As cells move through G2/M, the fraction of cells staining for phospho-S308 increased, peaking at $12 \mathrm{~h}$ after release (Supplementary Figure S5, see section on supplementary data at the end of this article). These results confirm that the AR is phosphorylated on S308 in G2/M.

Interestingly, when the phospho-S308 fraction was analyzed specifically in G2/M by gating on DNA content, approximately $14 \%$ of asynchronous LNCaP cells were positive for phospho-S308, whereas in cells enriched in G2/M with nocodazole, approximately $85 \%$ of cells were positive for phospho-S308 (Supplementary Figure S6, see section on supplementary data at the end of this article). Nocodazole treatment causes arrest of cells in prometaphase by interfering with microtubule polymerization. It is

Published by Bioscientifica Ltd. 


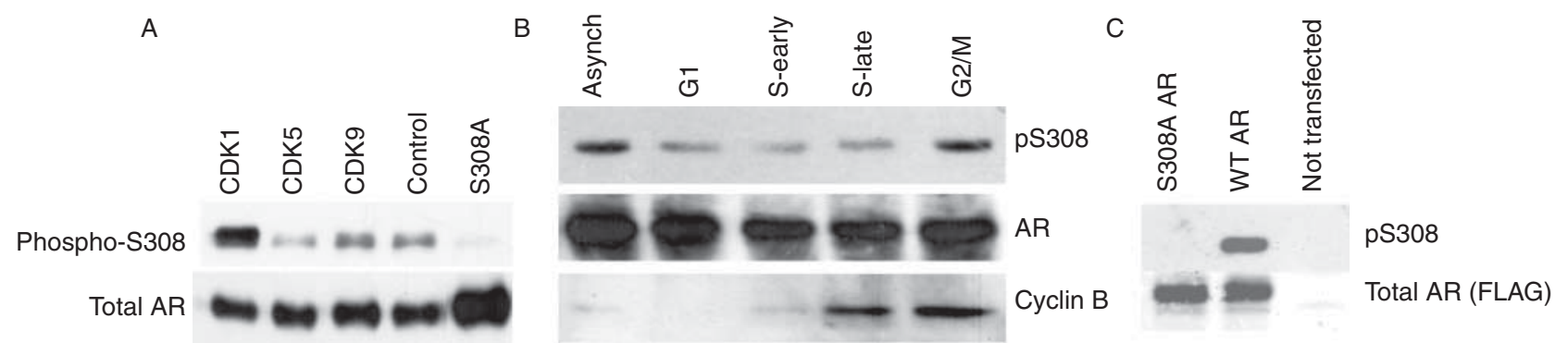

Figure 6

CDK1 phosphorylates the AR on S308, which is increased in G2/M. (A) Kinase assays were performed using purified CDKs and AR. Phosphorylation on $\mathrm{S} 308$ was assessed using phospho-S308 -specific antibodies. Control indicates the no-kinase control. S308A is an unphosphorylatable mutant. (B) LNCaP cells were stained with a DNA dye and sorted into cell cycle compartments by flow cytometry on the basis of DNA content. AR was immunoprecipitated from $1 \times 10^{6}$ cells in each cell cycle compartment and

interesting to note that the proportion of the phosphoS308-positive cells was higher in cells treated with nocodazole, indicating that AR is probably phosphorylated on S308 during early mitosis. To further delineate the cell cycle compartment where S308 phosphorylation takes place, cells were analyzed for the expression of the mitotic marker phospho-histone H3. Dual fluorescence analysis of phospho-S308 and phospho-histone H3 in LNCaP and C4-2 cells revealed that all phospho-S308-positive cells expressed the mitotic marker phospho-histone H3 (Fig. 7D, G, and K). Thus, our flow cytometry data indicate that the AR is phosphorylated on S308 in mitosis. Importantly, we found that AR S308 phosphorylation is hormone-independent. To assess the effect of a hormone on S308 phosphorylation, LNCaP cells were treated with a range of doses of DHT, $0.01-10 \mathrm{~nm}$, in steroid-free media for 2 and $16 \mathrm{~h}$ and the phospho-S308 signal was analyzed by using flow cytometry. As shown in Table 1, hormone treatment did not affect S308 phosphorylation.

\section{Pharmacological inhibition of CDK1 activity decreases S308 phosphorylation}

To further address whether S308 phosphorylation was mediated by CDK1, LNCaP cells were treated with either vehicle or the CDK1 selective inhibitor RO-3306 for $2 \mathrm{~h}$ (Vassilev et al. 2006). This relatively short time of treatment was chosen to minimize the affects of CDK1 inhibition on the cell cycle. Treatment led to a dramatic inhibition of S308 phosphorylation (Fig. 8). In vehicletreated cells, approximately $10.8 \%$ of cells were positive for phospho-S308 during G2/M (Fig. 8B and F), whereas in RO-3306-treated cells the phospho-S308-positive immunoblotted for total and phospho-S308 AR. The phospho-S308 signal is increased during G2/M when cyclin B1 protein levels from lysate are increased. (C) Phospho-S308 antibody specificity: COS7 cells transiently expressing either WT or S308A AR were treated with $1 \mathrm{nM} \mathrm{DHT}$ for $16 \mathrm{~h}$. AR was immunoprecipitated with anti-Flag antibody and blotted with anti-phospho-S308 (top panel) or anti-Flag antibody (lower panel).

population decreased to $2.3 \%$ (Fig. $8 \mathrm{D}$ and F). Treatment with the CDK1 inhibitor for $2 \mathrm{~h}$ did not change the cell cycle profile (Fig. 8E). These results, together with the results of the in vitro kinase assay and the results showing an increase in S308 phosphorylation in phospho-histone H3-positive cells, indicate that CDK1 phosphorylates the AR on S308 during mitosis.

\section{AR S308 phosphorylation regulates AR localization during mitosis}

Our confocal and ImageStream results indicate that AR localization changes during mitosis. We proposed the hypothesis that CDK1 phosphorylation of S308 in mitosis regulates this change in AR localization. To address this hypothesis, AR localization during the cell cycle was analyzed using a combination of phospho-S308-specific and three different anti-total-AR antibodies using confocal microscopy. One of the anti-total-AR antibodies we used, M441, is a mouse monoclonal antibody developed against AR amino acids 299-315 and thus contains the phosphosite S308. Importantly, M441 only recognizes nonphosphorylated S308 AR; phosphorylation on S308 blocks M441 binding (Supplementary Figure S7, see section on supplementary data at the end of this article). The antitotal-AR antibody AR21 is a rabbit polyclonal antibody raised against AR amino acids 1-21. AR21 recognizes both phosphorylated and non-phosphorylated S308 AR (Supplementary Figure S7). The immunostaining pattern of AR21 was identical to the pattern of a third anti-totalAR antibody (Supplementary Figure S8). Thus, these anti-total-AR antibodies can be used in conjunction with the phospho-S308-specific antibody to assess the

Published by Bioscientifica Ltd 
A

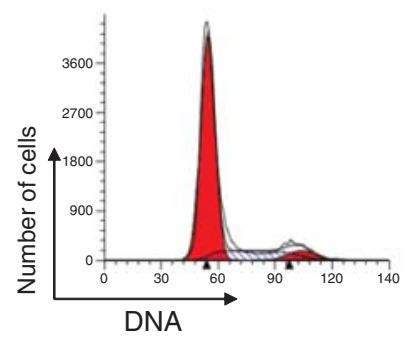

$\mathrm{H}$

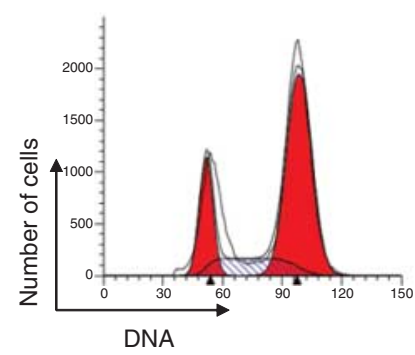

B

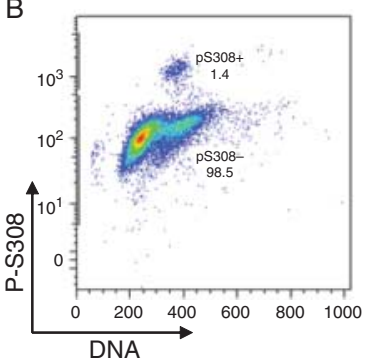

E

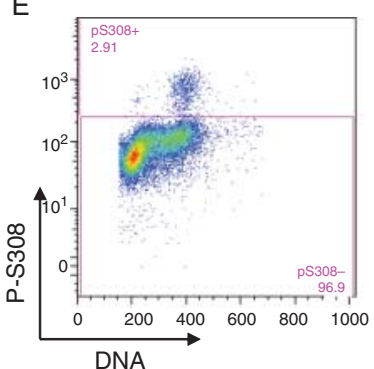

I

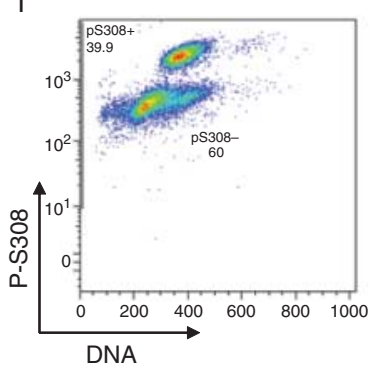

C

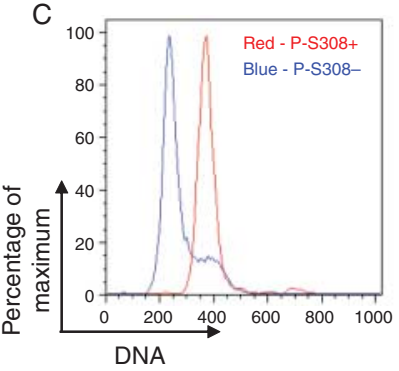

$\mathrm{F}$

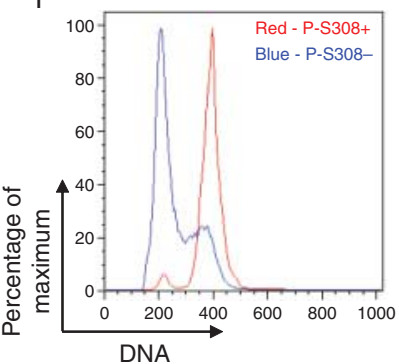

$\mathrm{J}$

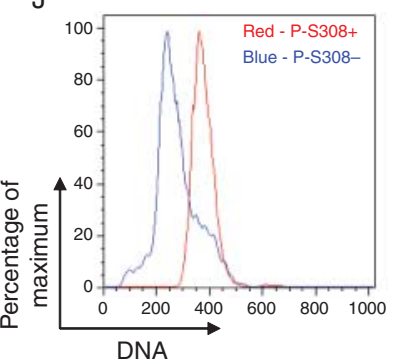

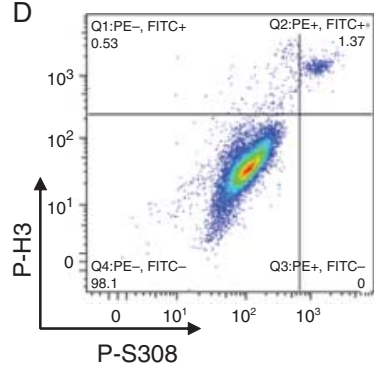

G

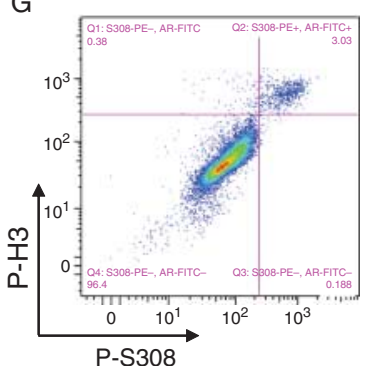

$\mathrm{K}$

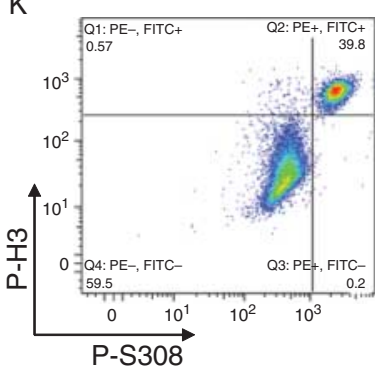

Figure 7

$A R$ is phosphorylated on $\$ 308$ during mitosis in prostate cancer cell lines. LNCaP and C4-2 cells were enriched in G2/M by aphidicolin/nocodazole treatment and processed for flow cytometry. $(\mathrm{A})$ and $(\mathrm{H})$, DNA profiles for asynchronous and chemically enriched G2/M LNCaP cells respectively. (B), (E) and (I), dual-fluorescence analyses of $\mathrm{S308}$ phosphorylation and DNA in asynchronous LNCaP, asynchronous C4-2, and G2/M enriched LNCaP cells

localization of phospho-S308 and non-phospho-S308 AR during interphase and mitosis.

Only mitotic, and not interphase, LNCaP cells had a phospho-S308 signal (Fig. 9A). The AR-negative PC3 cells did not show a phospho-S308 signal (Supplementary Figure 9A, see section on supplementary data at the end of this article). Interphase LNCaP cells, co-stained with M441 and AR21 total AR antibodies, had very similar nuclear patterns of staining and both of these antibodies showed very high co-localization with DNA, 92\% and 80\% co-localization with DNA respectively (Fig. 9B and C). In contrast, in mitotic cells these antibodies had very different staining patterns. M441 staining remained closely co-localized with chromosomes (86\% co-localization), whereas most of the AR signal was excluded from chromosomes when cells were stained with AR21 (only 31\% of the AR21 staining

respectively. Histograms show overlaid DNA profiles of phospho-S308-positive (red) and phospho-S308-negative (blue) populations for asynchronous LNCaP (C), asynchronous C4-2 (F), and G2/M-enriched LNCaP (J) cells. (D), (G) and (K) Results of dual-fluorescence analysis of $\mathrm{S} 308$ phosphorylation and the mitotic marker phospho-histone $\mathrm{H} 3$ staining in asynchronous $\mathrm{LNCaP}(\mathrm{D})$, asynchronous C4-2 (G), and G2/M-enriched LNCaP cells (K) respectively.

co-localized with DNA). Taken together, these results indicate that two forms of the AR are present during mitosis: phosphorylated S308 that is excluded from chromosomes and non-phosphorylated S308 that preserves chromosome association during mitosis.

\section{Discussion}

It is well established that the AR drives cell-cycle progression by regulating transcription of genes that control the G1-S transition, with substantive lines of cross-communication between the AR and the cell cycle machinery (Balk \& Knudsen 2008). However, little has been reported regarding the role of the AR in G2 or mitosis. In this study, we obtained data indicating that AR phosphorylation on $\mathrm{S} 308$ by CDK1 regulates $\mathrm{AR}$ 
Table 1 The effect of DHT on S308 phosphorylation

\begin{tabular}{|c|c|c|}
\hline DHT treatment & $\begin{array}{c}\text { Phospho-S308- } \\
\text { positive, } 2 \mathrm{~h}\end{array}$ & $\begin{array}{c}\text { Phospho-S308- } \\
\text { positive, } 16 \mathrm{~h}\end{array}$ \\
\hline Vehicle & $1.35 \pm 0.19$ & $1.68 \pm 0.42$ \\
\hline $0.01 \mathrm{~nm}$ & $1.45 \pm 0.48$ & $1.66 \pm 0.29$ \\
\hline $0.1 \mathrm{~nm}$ & $1.47 \pm 0.40$ & $1.57 \pm 0.33$ \\
\hline $1 \mathrm{~nm}$ & $1.75 \pm 0.61$ & $1.80 \pm 0.41$ \\
\hline $10 \mathrm{~nm}$ & $1.53 \pm 0.57$ & $1.81 \pm 0.53$ \\
\hline
\end{tabular}

LNCaP cells were grown in T-Media supplemented with $5 \%$ FBS. Before hormone treatment, media were replaced with RPMI supplemented with $5 \%$ CSS. The cells were treated with vehicle or DHT at concentrations ranging between 0.01 and $10 \mathrm{~nm}$ for 2 and $16 \mathrm{~h}$. The cells were fixed and processed for flow cytometry. S308 phosphorylation was assessed using phospho-S308-specific antibodies. Listed are the mean signal Is.E.M. Hormone treatment did not affect S308 phosphorylation: $P=0.979$ for $2 \mathrm{~h}$ and $P=0.992$ for $16 \mathrm{~h}$ using one-way ANOVA.

localization during mitosis and that this CDK1-mediated change in AR localization correlates with changes in AR transcriptional activity.

Other nuclear receptors also are reported to regulate and be regulated by the cell cycle, although the specific mechanisms and modalities of those interactions are different from those for the AR. Results of previous studies on the glucocorticoid receptor (GR), progesterone receptor (PR), estrogen receptor (ER), and thyroid receptor (TR) have indicated that transcriptional activity is regulated as a function of the cell cycle (Hsu \& DeFranco 1995, Maruvada et al. 2004, Narayanan et al. 2005, Dalvai \& Bystricky 2010). Results of several studies have indicated that GR-dependent transactivation is impaired in G2/Menriched nocodazole-treated cells when compared with asynchronous cells (Hsu et al. 1992, Hsu \& DeFranco 1995, Matthews et al. 2011). However, other results have indicated that GR transcriptional activity was similar in asynchronous and G2-enriched cells, and repressed during mitosis due to chromatin condensation (Hu etal. 1994, Abel et al. 2002). GR hormone binding was elevated during S-phase and G2 in Hela cells (Cidlowski \& Cidlowski 1982). The progesterone receptor (PR) transcriptional activity peaks during S-phase through the recruitment of cyclin/cdk2 to sites of PR transcription. The effects of chemical arrest of MCF-7 breast cancer cells indicated that several ER $\alpha$ target genes have higher expression during G1 compared with G2/M and that different selective estrogen modulators can either repress or stimulate expression of specific ER $\alpha$ target genes depending on the cell cycle phase (Dalvai \& Bystricky 2010). TR expression and transactivation were essentially absent during G1, increased during late $S$ and G2, and peaked during G2/M (Maruvada et al. 2004).

One previous study did address AR transcriptional activity during the cell cycle using reporter constructs in chemically arrested cells and 24-h hormone treatment (Martinez \& Danielsen 2002). Under those conditions, AR transcriptional activity was higher during $G_{0}$ and $S$ and reduced during the $\mathrm{G} 1 / \mathrm{S}$ transition and these transcriptional effects paralleled changes in AR expression; however, the G2/M cell cycle compartment was not examined. In our study, we used a brief 2-h change in charcoal-stripped media and androgen stimulus to assess androgen-induced transcription of the endogenous AR target genes SGK, SNAI2,
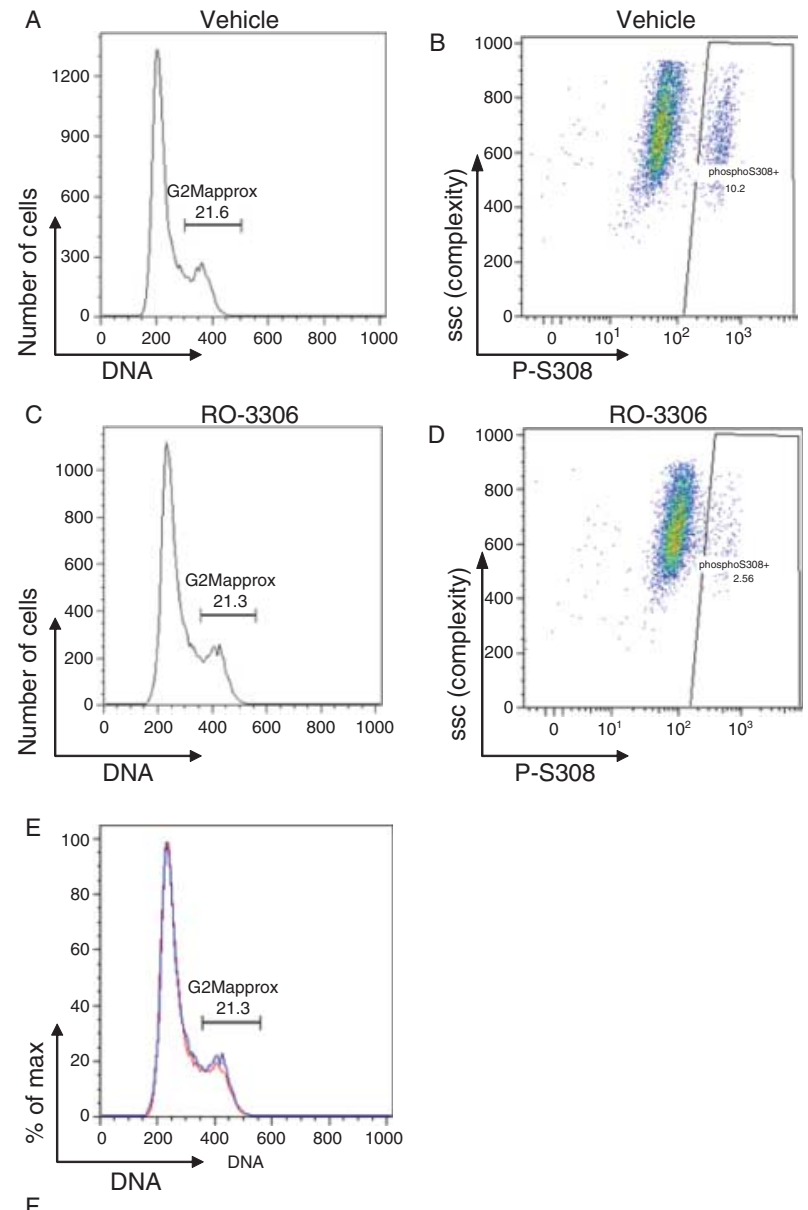

$\mathrm{F}$

\begin{tabular}{cc}
\hline Treatment & $\begin{array}{c}\text { Phospho-S308-positive during G2M, } \\
\text { Mean } \pm \text { S.E.M. }\end{array}$ \\
\hline DMSO & $10.76 \pm 1.39$ \\
RO-3306 & $2.31 \pm 0.58$ \\
\hline
\end{tabular}

\section{Figure 8}

Pharmacological inhibition of CDK1 results in a decrease in S308 phosphorylation. LNCaP cells were treated with either vehicle (A and B) or RO-3306 ( $C$ and D) for $2 \mathrm{~h}$ and phospho-S308 signal was analyzed by flow cytometry. Histograms (A) and (C) show DNA profiles for vehicle- (A) and RO-3306-treated (C) cells; gating on G2/M phase is shown. (B) and (D) show phospho-S308 signals in G2/M cells in vehicle- and RO-3306-treated cells respectively. (E) shows overlaid DNA profiles of RO-3306-treated (red) and vehicle-treated (blue) cells. (F) Table showing quantitation of S308 phosphorylation in RO-3306-treated and vehicle-treated cells, $n=3$. 
A
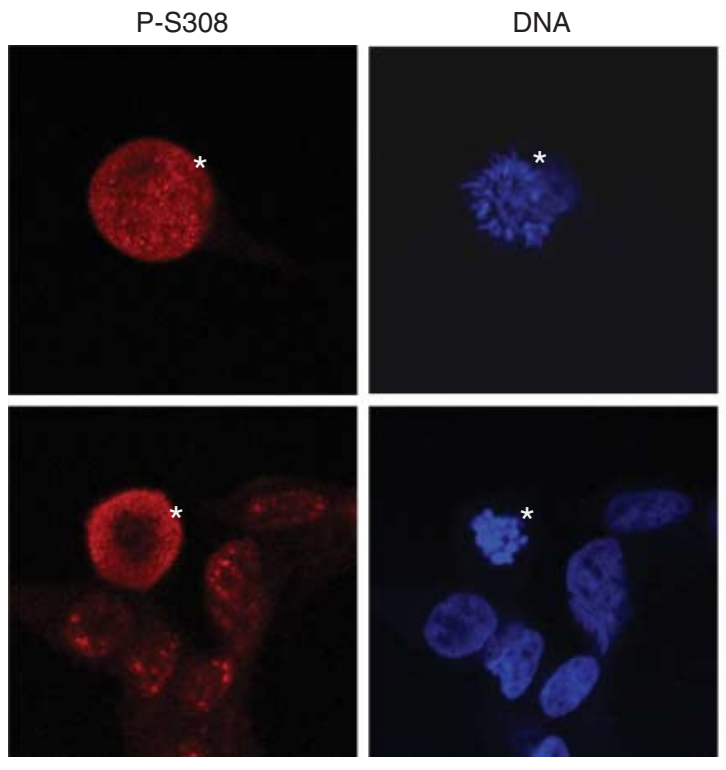
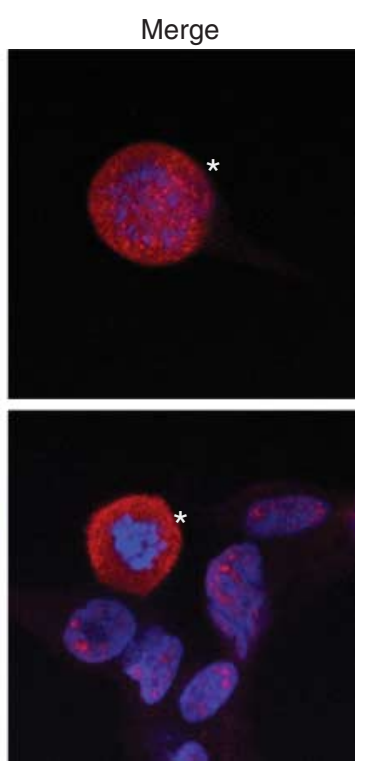

B
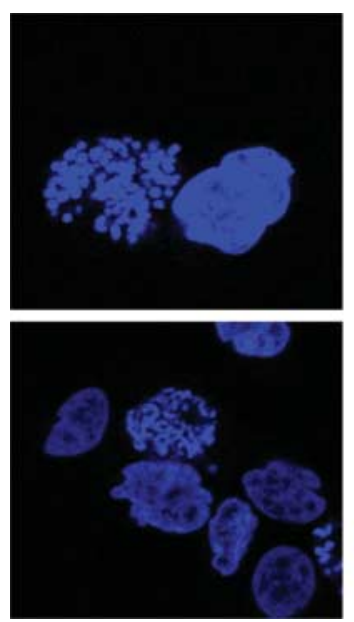

AR-M441 Ab
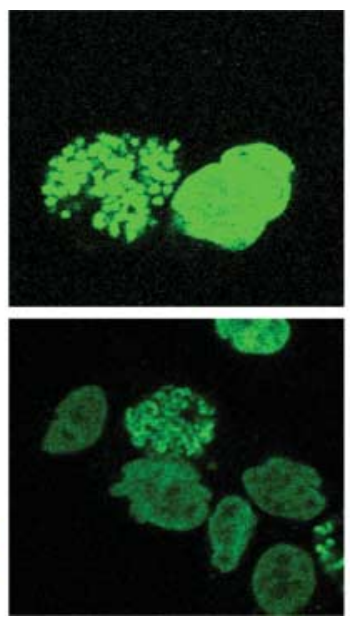

AR-AR21 Ab
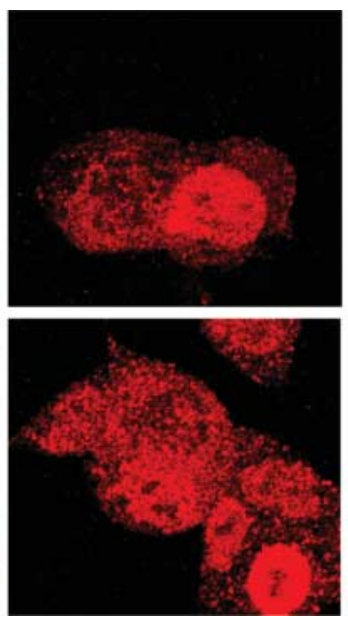

Merge
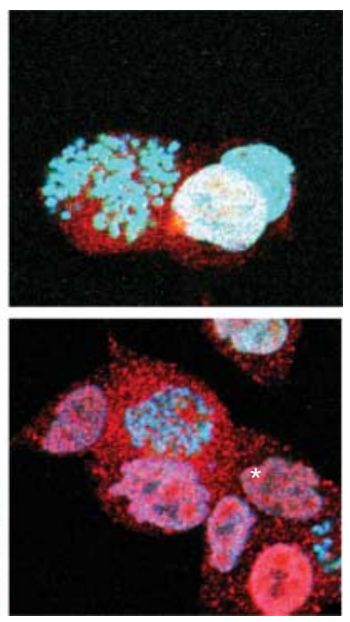

C

Anti-total AR

\section{Co-localization with DNA}

Mitotic

\begin{tabular}{ccc} 
& Interphase & Mitotic \\
\hline AR21 & $80 \pm 3.6$ & $31 \pm 2.6$ \\
\hline M441 & $92 \pm 4.1$ & $86 \pm 4.4$ \\
\hline
\end{tabular}

\section{Figure 9}

P-S308 signal is excluded from chromatin in LNCaP cells. (A) LNCaP cells were enriched in mitosis, fixed and stained with phospho-S308-specific antibody. P-S308 signal is present only in mitotic cells (mitotic cells are indicated by asterisks). (B) LNCaP cells were co-stained with two different

and TMPRSS 2 in different cell cycle compartments by FACS and found that androgen-stimulated AR transactivation is highest during G1 and essentially abrogated during G2/M. We did not observe differences in AR expression during the
anti-AR antibodies (M441 and AR21) targeting different epitopes. (C) Table showing quantitation of co-localization of the AR staining with DNA using the two different anti-AR antibodies in interphase and mitotic LNCaP cells.

cell cycle (see Fig. 2). There are many differences between our study and that of Martinez \& Danielsen (2002), including our use of human cell lines, endogenous gene targets, and asynchronous sorted cells, that may account for the different

Published by Bioscientifica Ltd. 
observations of AR levels during the cell cycle. Interestingly, when AR localization of exogenous GFP-tagged AR in response to hormone was examined, increased nuclear localization was observed in G2 cells (Szafran et al. 2008). This result indicates that AR transcriptional activity would be highest during G2. Our study only examined a small subset of AR target genes. To date, no published study, to our knowledge, has examined the global cell-cycle-dependent gene expression profile of the AR, or any other steroid hormone receptor. Thus, the increased hormone-induced AR nuclear localization during G2 may lead to increased transactivation of G2-specific AR target genes.

Results of an earlier study analyzing the effect of the cell cycle on AR protein expression indicated that AR protein expression was lost during mitosis and that the AR functions as a mitotic licensing factor (Litvinov et al. 2006). However, this is in contrast to the results of our experiments reported here as well as the results of studies by other groups showing AR expression during mitosis (Kumar et al. 2008). In our study, we detected AR expression throughout the cell cycle. Kumar et al. (2008) showed that overexpression of GFP-tagged AR in COS1 cells led to agonist-mediated docking of AR onto mitotic chromatin. The association of AR with chromatin was dependent on the method used for fixation/permeabilization, where one method provided evidence of endogenous hormonebound AR associated with mitotic chromatin and others showed the majority of the AR was excluded from chromatin. In our studies, different fixation/permeabilization methods did not reveal differences in AR localization or phosphorylation (data not shown). In further support of AR expression during mitosis, results of other studies have indicated that CDK1 activity, which peaks during $\mathrm{G} 2 / \mathrm{M}$, stabilizes the AR (Chen et al. 2006). One possible explanation for the discrepancy among these reports of AR expression during mitosis is that the AR is degraded late in mitosis. However, using ImageStream analysis we observed endogenous AR during all mitotic phases, including telophase, consistent with AR expression being maintained throughout mitosis. Another possibility is the method used to analyze the flow cytometry data (Litvinov et al. 2006), which could lead to alternative conclusions.

While uncommon, there is a precedent for transcription factor association and activity during mitosis even though chromatin favors a highly condensed state and global transcription during mitosis is silenced (Gottesfeld \& Forbes 1997). Most basal transcription factors, RNA polymerases, and sequence-specific DNA-binding proteins and histone modifying enzymes are absent from condensed mitotic chromosomes. However, evidence indicates that certain transcription factors remain bound to mitotic condensed chromosomes (Burke et al. 2005, Saradhi et al. 2005, Yan et al. 2006, Young et al. 2007). For example, the zinc-finger protein CTCF and the lineage-specific transcription factor RUNX2 are implicated in the maintenance of epigenetic marks during mitosis (Burke et al. 2005, Young et al. 2007) and the forkhead FoxI1 transcription factor is implicated in organization of mitotic chromatin structure (Yan et al. 2006).

Our data are in general agreement with results of studies of GR localization demonstrating GR nuclear localization throughout interphase and exclusion from the nucleus during mitosis (Hsu et al. 1992, Matthews et al. 2011). In these studies, it was suggested that recycled GRs do not re-enter the nucleus in G2-synchronized cells (Hsu et al. 1992). GR localization during mitosis did not change with dexamethasone treatment, which is similar to our finding that hormone treatment did not alter AR localization during mitosis. Interestingly, dexamethasone-induced GR transactivation was blocked in G2 synchronized cells, but not in asynchronous cells, although the number of receptors remained the same in the two populations. This latter finding is consistent with our observations of AR transcriptional activity decreasing during $\mathrm{G} 2 / \mathrm{M}$, although $\mathrm{AR}$ protein levels remain unchanged throughout the cell cycle. Interestingly, the binding of a nuclear receptor family member pregnane and xenobiotic receptor (PXR) to mitotic chromatin during all mitotic phases has been reported for an overexpression system, although the significance of that association was not elucidated (Saradhi et al. 2005).

In this study, we show that AR phosphorylated on S308 is excluded from chromosomes and that nonphosphorylated S308 preserves chromosome association during mitosis in a hormone-independent manner. AR phosphorylation on S308 is coincident with peak CDK1 activity. Previous work performed at our laboratory and others has collectively demonstrated that CDK1 interacts with the AR and can phosphorylate AR S81 (Chen et al. 2006, 2012, Gordon et al. 2010) and nocodazole-arrested cells in G2/M have elevated AR S81 phosphorylation (Chen et al. 2006, 2012) although our results indicate that CDK9 is the major AR S81 kinase (Gordon et al. 2010) and it remains unclear under what biological conditions each of the reported CDKs phosphorylate the AR on S81. In experiments reported here we demonstrate that CDK1 phosphorylates the AR on $\mathrm{S} 308$ in vitro and that pharmacological inhibition of CDK1 activity dramatically decreases S308 phosphorylation in PCa cells. Interestingly, even though we previously observed an increase in S308 phosphorylation in response to hormone (Gioeli et al.

Published by Bioscientifica Ltd. 
2002), a thorough analysis of S308 phosphorylation in mitotic cells reported here indicates that S308 phosphorylation is hormone-independent. It is likely that the slight increase in AR S308 phosphorylation observed during phospho-peptide mapping studies were due to an increase in the percentage of mitotic cells resulting from hormone stimulation. Results of a previous study also indicated that S308 is phosphorylated by the CDK11 ${ }^{\mathrm{p} 58}$-cyclin D3 complex during $\mathrm{G} 2 / \mathrm{M}$ leading to repression of $\mathrm{AR}$ transcriptional activity (Zong et al. 2007). This is in agreement with the results described in our earlier publication (Gioeli et al. 2002) and parallels our observations described in this report in which AR transcriptional activity decreases coincidentally with S308 phosphorylation. Interestingly, results from one study have indicated that S308 phosphorylation correlates with patient survival (McCall et al. 2013), which is consistent with the results indicating that phosphorylation at this site may reduce AR transcriptional activity.

Interestingly, the GR is also phosphorylated in a cellcycle-dependent manner (Matthews et al. 2011). Results of studies of the GR indicate that G2-synchronized cells have a distinct phosphorylation pattern compared with asynchronous cells (Hsu et al. 1992), although comparison of HPLC phosphopeptide maps revealed no phospho-sitespecific differences in WCL2 cells synchronized in S-phase and G2/M (Hu et al. 1994). Overall GR phosphorylation is increased during G2/M compared with S-phase, whereas hormone-dependent induction is higher during S-phase and negligible during G2/M. GR phosphorylation on S203 and S211 occurred in a ligand-independent fashion during G2/M and ligand-independent GR transactivation required S211 phosphorylation (Matthews et al. 2011).

Our experiments provide evidence that the cell cycle affects AR function. On the basis of these results we propose a model in which the AR S308 phosphorylation by CDK1 results in the AR exclusion from mitotic chromatin thereby negatively regulating AR-dependent transcription. Using the results of this study it is possible to begin to integrate the complexity of cycling cells and signal transduction by kinases with AR biology.

\section{Supplementary data}

This is linked to the online version of the paper at http://dx.doi.org/10.1530/ ERC-14-0549.

\section{Declaration of interest}

The authors declare that there is no conflict of interest that could be perceived as prejudicing the impartiality of the research reported.

\section{Funding}

Grant support: National Cancer Institute (R01 CA124706 and R01 CA178338, D Gioeli), the Paul Mellon Urologic Cancer Institute (D Gioeli), the Farrow Fellowship (Y Koryakina), and Prostate Cancer Foundation Challenge Award (K E Knudsen).

\section{Acknowledgements}

The authors would like the thank Dr Huy Ta and Christopher McNair for critically reading the manuscript. They would also like to thank Joanne Lannigan and Mike Solga of the University of Virginia Flow Cytometry Core Facility for outstanding technical support.

\section{References}

Abel GA, Wochnik GM, Rüegg J, Rouyer A, Holsboer F \& Rein T 2002 Activity of the GR in G2 and mitosis. Molecular Endocrinology 16 1352-1366. (doi:10.1210/mend.16.6.0842)

Balk SP \& Knudsen KE 2008 AR, the cell cycle, and prostate cancer. Nuclear Receptor Signaling 6 e001. (doi:10.1621/nrs.06001)

Burd CJ, Petre CE, Morey LM, Wang Y, Revelo MP, Haiman CA, Lu S, Fenoglio-Preiser CM, Li J, Knudsen ES et al. 2006 Cyclin D1b variant influences prostate cancer growth through aberrant androgen receptor regulation. PNAS 103 2190-2195. (doi:10.1073/pnas.0506281103)

Burke LJ, Zhang R, Bartkuhn M, Tiwari VK, Tavoosidana G, Kurukuti S, Weth C, Leers J, Galjart N, Ohlsson R et al. 2005 CTCF binding and higher order chromatin structure of the H19 locus are maintained in mitotic chromatin. EMBO Journal 24 3291-3300. (doi:10.1038/sj. emboj.7600793)

Chen S, Xu Y, Yuan X, Bubley GJ \& Balk SP 2006 Androgen receptor phosphorylation and stabilization in prostate cancer by cyclin-dependent kinase 1. PNAS 103 15969-15974. (doi:10.1073/pnas.0604193103)

Chen S, Gulla S, Cai C \& Balk SP 2012 Androgen receptor serine 81 phosphorylation mediates chromatin binding and transcriptional activation. Journal of Biological Chemistry 287 8571-8583. (doi:10.1074/ jbc.M111.325290)

Cidlowski JA \& Cidlowski NB 1982 Glucocorticoid receptors and the cell cycle: evidence that the accumulation of glucocorticoid receptors during the $S$ phase of the cell cycle is dependent on ribonucleic acid and protein synthesis. Endocrinology 110 1653-1662. (doi:10.1210/endo-110-5-1653)

Comstock CE \& Knudsen KE 2007 The complex role of AR signaling after cytotoxic insult: implications for cell-cycle-based chemotherapeutics. Cell Cycle 6 1307-1313. (doi:10.4161/cc.6.11.4353)

Dalvai M \& Bystricky K 2010 Cell cycle and anti-estrogen effects synergize to regulate cell proliferation and ER target gene expression. PLOS ONE 5 e11011. (doi:10.1371/journal.pone.0011011)

George D \& Moul JW 2011 Emerging treatment options for patients with castration-resistant prostate cancer. Prostate 72 338-349. (doi:10.1002/ pros.21435)

Gioeli D, Ficarro SB, Kwiek JJ, Aaronson D, Hancock M, Catling AD, White FM, Christian RE, Settlage RE, Shabanowitz J et al. 2002 Androgen receptor phosphorylation. Regulation and identification of the phosphorylation sites. Journal of Biological Chemistry $\mathbf{2 7} 7$ 29304-29314. (doi:10.1074/jbc.M204131200)

Gordon V, Bhadel S, Wunderlich W, Zhang J, Ficarro SB, Mollah SA, Shabanowitz J, Hunt DF, Xenarios I, Hahn WC et al. 2010 CDK9 regulates AR promoter selectivity and cell growth through serine 81 phosphorylation. Molecular Endocrinology 24 2267-2280. (doi:10.1210/ me.2010-0238)

Gottesfeld JM \& Forbes DJ 1997 Mitotic repression of the transcriptional machinery. Trends in Biochemical Sciences 22 197-202. (doi:10.1016/ S0968-0004(97)01045-1) 
Güttinger S, Laurell E \& Kutay U 2009 Orchestrating nuclear envelope disassembly and reassembly during mitosis. Nature Reviews. Molecular Cell Biology 10 178-191. (doi:10.1038/nrm2641)

Hsu SC \& DeFranco DB 1995 Selectivity of cell cycle regulation of glucocorticoid receptor function. Journal of Biological Chemistry $\mathbf{2 7 0}$ 3359-3364.

Hsu SC, Qi M \& DeFranco DB 1992 Cell cycle regulation of glucocorticoid receptor function. EMBO Journal 11 3457-3468.

Hu JM, Bodwell JE \& Munck A 1994 Cell cycle-dependent glucocorticoid receptor phosphorylation and activity. Molecular Endocrinology $\mathbf{8}$ 1709-1713. (doi:10.1210/mend.8.12.7708059)

Joseph JD, Lu N, Qian J, Sensintaffar J, Shao G, Brigham D, Moon M, Maneval EC, Chen I, Darimont B et al. 2013 A clinically relevant androgen receptor mutation confers resistance to second-generation antiandrogens enzalutamide and ARN-509. Cancer Discovery 3 1020-1029. (doi:10.1158/2159-8290.CD-13-0226)

Knudsen KE 2006 The cyclin D1b splice variant: an old oncogene learns new tricks. Cell Division 1 15. (doi:10.1186/1747-1028-1-15)

Knudsen KE, Arden KC \& Cavenee WK 1998 Multiple $\mathrm{G}_{1}$ regulatory elements control the androgen-dependent proliferation of prostatic carcinoma cells. Journal of Biological Chemistry 273 20213-20222. (doi:10.1074/jbc.273.32.20213)

Knudsen KE, Cavenee WK \& Arden KC 1999 D-type cyclins complex with the androgen receptor and inhibit its transcriptional transactivation ability. Cancer Research 59 2297-2301.

Koryakina Y, Jones SM, Cornett LE, Seely K, Brents L, Prather PL, Kofman A $\&$ Kurten RC 2012 Effects of the $\beta$-agonist, isoprenaline, on the down-regulation, functional responsiveness and trafficking of $\beta_{2}$-adrenergic receptors with N-terminal polymorphisms. Cell Biology International 36 1171-1183. (doi:10.1042/CBI20120134)

Koryakina Y, Ta HQ \& Gioeli D 2014 Androgen receptor phosphorylation: biological context and functional consequences. Endocrine-Related Cancer 21 T131-T145. (doi:10.1530/ERC-13-0472)

Kumar S, Chaturvedi NK, Kumar S \& Tyagi RK 2008 Agonist-mediated docking of androgen receptor onto the mitotic chromatin platform discriminates intrinsic mode of action of prostate cancer drugs. Biochimica et Biophysica Acta 1783 59-73. (doi:10.1016/j.bbamcr.2007. 11.002)

Lim JT, Mansukhani M \& Weinstein IB 2005 Cyclin-dependent kinase 6 associates with the androgen receptor and enhances its transcriptional activity in prostate cancer cells. PNAS 102 5156-5161. (doi:10.1073/ pnas.0501203102)

Litvinov IV, Vander Griend DJ, Antony L, Dalrymple S, De Marzo AM, Drake CG \& Isaacs JT 2006 Androgen receptor as a licensing factor for DNA replication in androgen-sensitive prostate cancer cells. PNAS 103 15085-15090. (doi:10.1073/pnas.0603057103)

Lu S, Liu M, Epner DE, Tsai SY \& Tsai MJ 1999 Androgen regulation of the cyclin-dependent kinase inhibitor p21 gene through an androgen response element in the proximal promoter. Molecular Endocrinology 13 376-384. (doi:10.1210/mend.13.3.0254)

Martinez ED \& Danielsen M 2002 Loss of androgen receptor transcriptional activity at the $\mathrm{G}_{1} / \mathrm{S}$ transition. Journal of Biological Chemistry $\mathbf{2 7 7}$ 29719-29729. (doi:10.1074/jbc.M112134200)

Maruvada P, Dmitrieva NI, East-Palmer J \& Yen PM 2004 Cell cycledependent expression of thyroid hormone receptor- $\beta$ is a mechanism for variable hormone sensitivity. Molecular Biology of the Cell $\mathbf{1 5}$ 1895-1903. (doi:10.1091/mbc.E03-09-0636)

Matthews L, Johnson J, Berry A, Trebble P, Cookson A, Spiller D, Rivers C, Norman M, White M \& Ray D 2011 Cell cycle phase regulates glucocorticoid receptor function. PLoS ONE 6 e22289. (doi:10.1371/ journal.pone.0022289)

McCall P, Adams CE, Willder JM, Bennett L, Qayyum T, Orange C, Underwood MA \& Edwards J 2013 Androgen receptor phosphorylation at serine 308 and serine 791 predicts enhanced survival in castrate resistant prostate cancer patients. International Journal of Molecular Sciences 14 16656-16671. (doi:10.3390/ijms140816656)

Narayanan R, Adigun AA, Edwards DP \& Weigel NL 2005 Cyclin-dependent kinase activity is required for progesterone receptor function: novel role for cyclin A/Cdk2 as a progesterone receptor coactivator. Molecular and Cellular Biology 25 264-277. (doi:10.1128/MCB.25.1.264-277.2005)

Petre CE, Wetherill YB, Danielsen M \& Knudsen KE 2002 Cyclin D1: mechanism and consequence of androgen receptor co-repressor activity. Journal of Biological Chemistry 277 2207-2215. (doi:10.1074/ jbc.M106399200)

Reutens AT, Fu M, Wang C, Albanese C, McPhaul MJ, Sun Z, Balk SP, Janne OA, Palvimo JJ \& Pestell RG 2001 Cyclin D1 binds the androgen receptor and regulates hormone-dependent signaling in a p300/CBPassociated factor (P/CAF)-dependent manner. Molecular Endocrinology 15 797-811. (doi:10.1210/mend.15.5.0641)

Saradhi M, Sengupta A, Mukhopadhyay G \& Tyagi RK 2005 Pregnane and xenobiotic receptor (PXR/SXR) resides predominantly in the nuclear compartment of the interphase cell and associates with the condensed chromosomes during mitosis. Biochimica et Biophysica Acta 1746 85-94. (doi:10.1016/j.bbamcr.2005.10.004)

Szafran AT, Szwarc M, Marcelli M \& Mancini MA 2008 Androgen receptor functional analyses by high throughput imaging: determination of ligand, cell cycle, and mutation-specific effects. PLOS ONE 3 e3605. (doi:10.1371/journal.pone.0003605)

Van Hooser A, Goodrich DW, Allis CD, Brinkley BR \& Mancini MA 1998 Histone $\mathrm{H} 3$ phosphorylation is required for the initiation, but not maintenance, of mammalian chromosome condensation. Journal of Cell Science 111 3497-3506.

Vassilev LT, Tovar C, Chen S, Knezevic D, Zhao X, Sun H, Heimbrook DC \& Chen L 2006 Selective small-molecule inhibitor reveals critical mitotic functions of human CDK1. PNAS 103 10660-10665. (doi:10.1073/ pnas.0600447103)

Whitworth H, Bhadel S, Ivey M, Conaway M, Spencer A, Hernan R, Holemon H \& Gioeli D 2012 Identification of kinases regulating prostate cancer cell growth using an RNAi phenotypic screen. PLoS ONE 7 e38950. (doi:10.1371/journal.pone.0038950)

Xu Y, Chen SY, Ross KN \& Balk SP 2006 Androgens induce prostate cancer cell proliferation through mammalian target of rapamycin activation and post-transcriptional increases in cyclin D proteins. Cancer Research 66 7783-7792. (doi:10.1158/0008-5472.CAN-05-4472)

Yamamoto A, Hashimoto Y, Kohri K, Ogata E, Kato S, Ikeda K \& Nakanishi M 2000 Cyclin $\mathrm{E}$ as a coactivator of the androgen receptor. Journal of Cell Biology 150 873-880. (doi:10.1083/jcb.150.4.873)

Yan J, Xu L, Crawford G, Wang Z \& Burgess SM 2006 The forkhead transcription factor FoxI1 remains bound to condensed mitotic chromosomes and stably remodels chromatin structure. Molecular and Cellular Biology 26 155-168. (doi:10.1128/MCB.26.1.155-168.2006)

Young DW, Hassan MQ, Yang X-Q, Galindo M, Javed A, Zaidi SK, Furcinitti P, Lapointe D, Montecino M, Lian JB et al. 2007 Mitotic retention of gene expression patterns by the cell fate-determining transcription factor Runx2. PNAS 104 3189-3194. (doi:10.1073/pnas.0611419104)

Zong H, Chi Y, Wang Y, Yang Y, Zhang L, Chen H, Jiang J, Li Z, Hong Y, Wang $\mathrm{H}$ et al. 2007 Cyclin D3/CDK11 ${ }^{\mathrm{p} 58}$ complex is involved in the repression of androgen receptor. Molecular and Cellular Biology 27 7125-7142. (doi:10.1128/MCB.01753-06)

Received in final form 28 January 2015

Accepted 13 February 2015

Made available online as an Accepted Preprint

17 February 2015 http://erc.endocrinology-journals.org

DOI: 10.1530/ERC-14-0549
(C) 2015 Society for Endocrinology Printed in Great Britain
Published by Bioscientifica Ltd 